\title{
RECENT RF RESULTS FROM THE MUCOOL TEST AREA*
}

\author{
J. Norem ${ }^{\#}$, ANL, Argonne, IL, 60439 USA \\ A. Bross, A. Moretti, Z. Qian, FNAL, Batavia, IL 60510 USA \\ D. Huang, Y. Torun, IIT, Chicago, IL 60616 USA \\ R. Rimmer, JLab, Newport News, VA, 23606, USA \\ D. Li, M. Zisman, LBNL, Berkeley, CA, 94720 USA
}

\section{Abstract}

The MuCool Experiment has been continuing to take data with 805 and $201 \mathrm{MHz}$ cavities in the MuCool Test Area. The system uses rf power sources from the Fermilab Linac. Although the experimental program is primarily aimed at the Muon Ionization Cooling Experiment (MICE), we have been studying the dependence of rf limits on frequency, cavity material, high magnetic fields, gas pressure, coatings, etc. with the general aim of understanding the basic mechanisms involved. The $201 \mathrm{MHz}$ cavity, essentially a prototype for the MICE experiment, was made using cleaning techniques similar to those employed for superconducting cavities and operates at its design field with very little conditioning.

\section{INTRODUCTION}

Our ultimate goal is the development of muon cooling systems for muon colliders and neutrino factories. We are continuing in the MTA an experimental program that was begun at Fermilab in 2001. This effort has the general aim of understanding and improving the state of the art for low frequency rf. as part of the MUCOOL program, and with the specific goal of developing and testing $\mathrm{rf}$ systems relevant to the Muon Ionization Cooling Experiment (MICE) at RAL [1].

Since muons do not interact strongly, rf systems used in muon cooling use thin Be windows at the ends of pillbox cavities to increase the accelerating field without significantly scattering or heating the beam. Although most of the cooling will be done in high magnetic fields, our $5 \mathrm{~T}$ solenoid is only large enough to hold the 805 MHz cavity [2].

\section{MHZ CAVITY}

The $805 \mathrm{MHz}$ cavity was used for curved window tests and measurements of buttons.

\section{Curved Windows}

In previous operation of the MUCOOL pillbox cavity with thin flat $\mathrm{Be}$ windows we found that the resonant cavity frequency at high cavity fields was pulse-to-pulse unstable by $+/-30 \mathrm{kHz}$. This instability was due to the EM impulse and instantaneous rf heating and subsequent movement and oscillation of the window. Under the

\footnotetext{
*Work supported by USDOE/HEP

"norem@anl.gov
}

influence of the EM impulse and instantaneous rf heating the windows deformed and oscillated in unpredictable ways. However, this instability went away when the magnetic field was turned on due to Eddy current braking effect.

Flat windows were replaced by curved windows designed by W. Lau and S. Yang of Oxford University that were thinner $(0.38 \mathrm{~mm})$, with better mechanical behavior, since rf heating strained the windows in a known way, and predictable frequency detuning without frequency pulse to pulse oscillations. The windows were installed so that the curvature on both windows went the same way, the actual detuning was negligible, and the cavity operation was found to be pulse to pulse frequency stable.

\section{Conditioning with Magnetic Field}

Conditioning with magnetic field has been one of the fundamental questions of muon cooling. In order to verify that the cavity was operating near the maximum asymptotic limit we wanted to operate the cavity with and without fields, and for a long conditioning period with B field. We ran for about one week with a $2.5 \mathrm{~T}$ solenoidal field and the accelerating field continued to decrease or hold essentially steady with time during this period.

The following plot shows the development of the operating field with time. We correct the measured cavity field, $E$, using the current radiation field intensity, $R$, to produce the equivalent field, $E_{\text {equiv }}=E_{\text {meas }} / R^{15}$, that would be measured with if the cavity was operated at a constant radiation flux $(1 \mathrm{mr} / \mathrm{hr})$. Fig. 1 shows the result of these tests. Following the initial turn on of the B field at $2 \mathrm{~T}$, the operating field of cavity dropped by about $30 \%$, (red triangles). Turning off the field immediately raised the operating gradient (blue circles), but turning it off again continued to approach a lower asymptotic limit. When

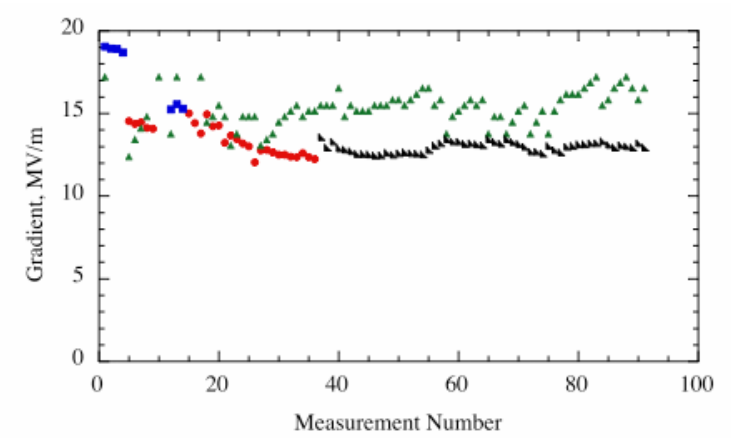

Figure 1: Conditioning for a week with solenoidal field. 
the pulse length was shortened to $20 \mu$ s the decline in the operating field seemed to level off (black triangles). The uncorrected data is shown as green triangles.

\section{Button Tests}

We have begun a program of testing different materials and surface treatments using small buttons that can be quickly removed from the cavity. We have a buttons of a number of materials and coatings and we are trying to test these using the Field Emission Microscope at JLab.

After operation in the cavity for a period of about 2 months, with magnetic fields as high as $4 \mathrm{~T}$ and electric fields on the order of $30 \mathrm{MV} / \mathrm{m}$, the cavity copper button shown in Fig. 2 shows the signs of many sparks, none of which seems to have been particularly damaging.

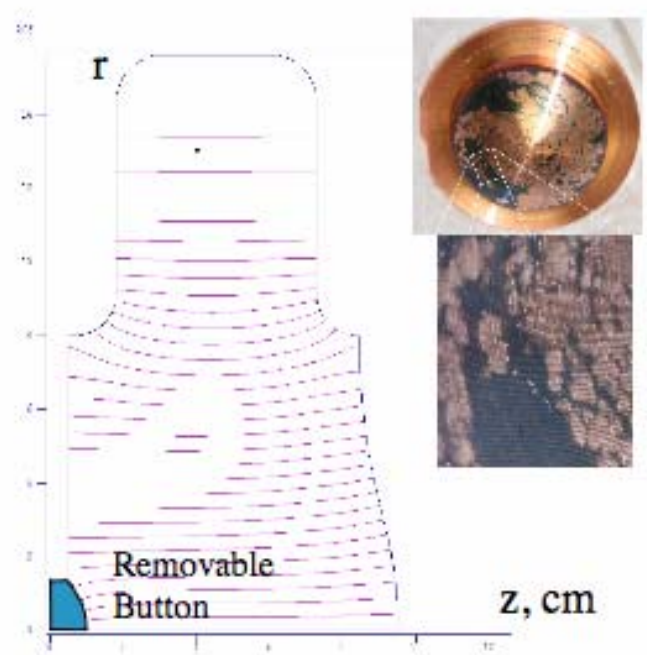

Figure 2: The E field of the button and pictures of a TiN coated copper button.

\section{Maximum gradient}

Extensive testing of maximum field that could be produced as a function of magnetic field is being done systematically with a variety of buttons and some preliminary results are available. These are shown in Fig. 3. We plot the maximum accelerating field reached in a short conditioning period.

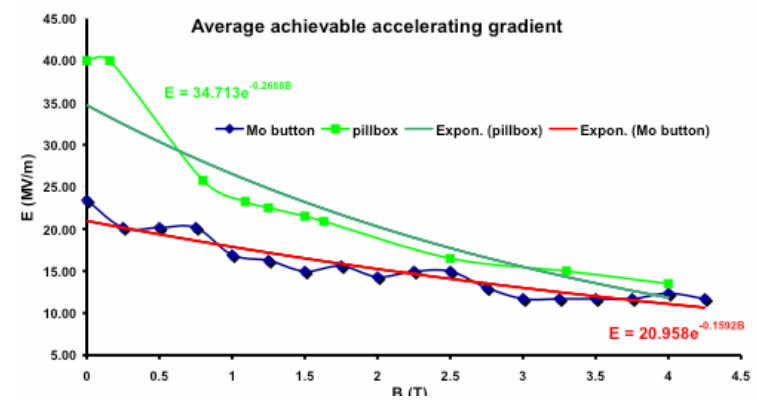

Figure 3: Maximum Accelerating field vs. B.

\section{MHZ CAVITY}

The $201 \mathrm{MHz}$ cavity is essentially a prototype cavity for the MICE experiment. The size is required to capture muon bunches of dimensions $\sim 30 \mathrm{~cm}$, and the structures are required to operate in a magnetic field. The construction of this cavity has been extensively documented [3] .

\section{Flat / Curved Window}

Like the $805 \mathrm{MHz}$ cavity we started operation of the 201 $\mathrm{MHz}$ cavity with flat windows, in this case quite thick $(11 \mathrm{~cm})$ to avoid deflection. The flat windows were recently replaced with curved windows again designed by W. Lau and S. Wang of Oxford. When the windows were installed in the cavity the curvature was aligned parallel, however the frequency shift caused by the change in geometry was significant, from 199.220 to $198.841 \mathrm{MHz}$, which seemed to push the power supply beyond its normal tuning range, requiring mechanical adjustment of the cavity to reach full power. The frequency change was predicted by Superfish simulation of the geometry changes in the cavity from flat to curved windows.

This cavity was made very carefully at JLab using surface polishing techniques usually reserved for superconducting systems. The result of this was that the structure reached $16 \mathrm{MV} / \mathrm{m}$ very quickly, with just a few breakdown events, and operated at fields up to $18 \mathrm{MV} / \mathrm{m}$ without any problems.

In order to replace the thick copper windows with thin, curved Be windows, we covered the system with a portable clean room and used clean room techniques to remove and replace the windows. When we opened the cavity up we saw that the cavity had no visible arc pits or damage anywhere on the interior surface, and only two pits on the thick copper window. The copper was essentially undamaged. A photo of the cavity interior is shown in Fig. 4.

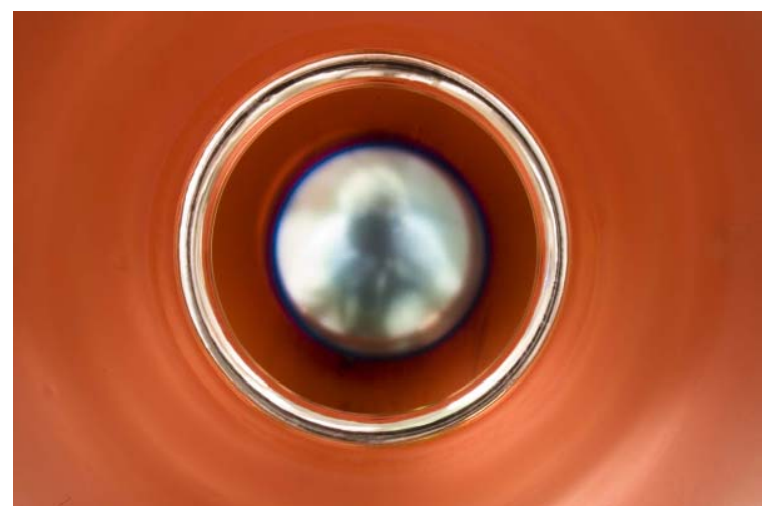

Figure 4: The interior of the $201 \mathrm{MHz}$ cavity.

The thin window was designed to expand in a known way and remain rigid during thermal cycling. Tests on the stability of this window are incomplete. The windows are $45 \mathrm{~cm} \mathrm{OD,} \mathrm{bonded} \mathrm{to} \mathrm{stiffener} \mathrm{rigs} \mathrm{at} \mathrm{the}$ circumference, with a bolt circle for mounting in the 
cavity The Be was TiN coated to eliminate multipactor. A photograph of the window is shown in Fig. 5.

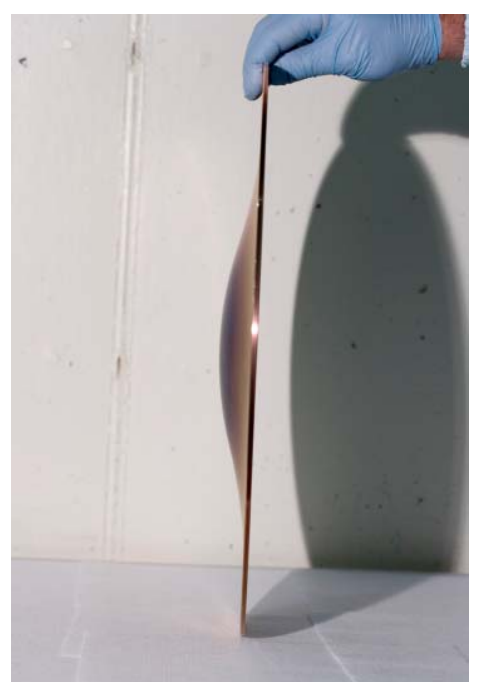

Figure 5: The Be window.

\section{Multipactor}

We see FN field emission as predicted, but also see (Fig. 6) multipactor resonances with $Q \sim 7$, which, in principle. could cause significant problems in the MICE spectrometer system. The resonance is seen at $5 \mathrm{MV} / \mathrm{m}$ in the fringe field of the solenoid operating at a field of 1.25T. Between the E14 Fowler-Nordheim emission and the resonance there is some rate during the rise and fall of the rf pulse, thus this would not contribute to the backgrounds in the MICE spectrometers. Multipactor should also not contribute to spectrometer backgrounds if it is possible to coat the interior of the cavity with TiN, which was done only for the windows.

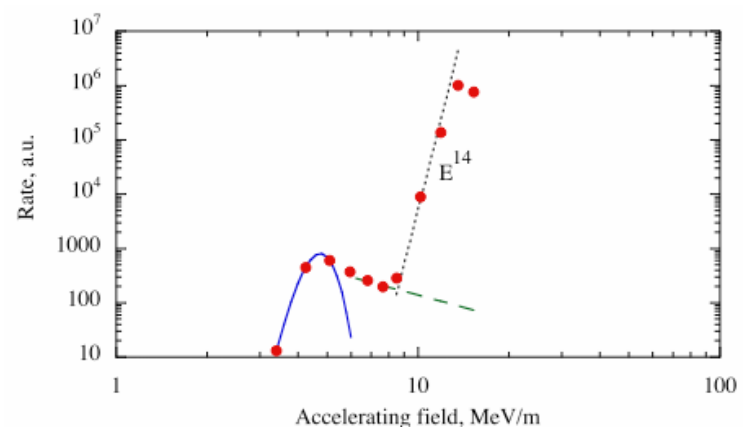

Figure 6: Higher than expected counting rate at low fields, presumably due to multipactor.

\section{FUTURE 201 MHZ PROGRAM}

In order to do useful tests of the $201 \mathrm{MHz}$ cavity in a magnetic field, we are constructing a coupling coil, which is large enough to enclose the large cavity. This will allow us to simulate and study the environment of the $\mathrm{rf}$ systems in the mice experiment.

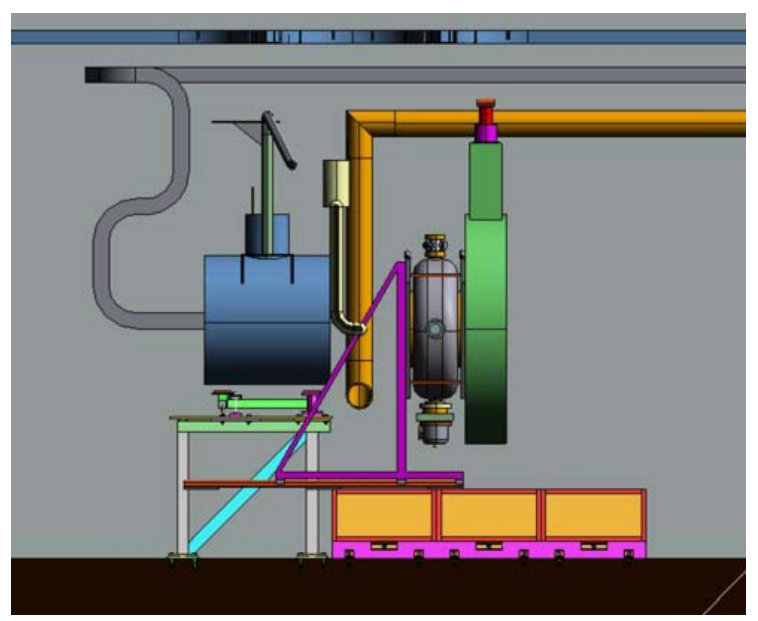

Figure 7: The small $5 \mathrm{~T}$ solenoid (blue), the $805 \mathrm{MHz}$ cavity (gray) and the coupling coil (green) which is under construction (schematic).

\section{ACKNOWLEDGEMENTS}

We would like to acknowledge the support of the USDOE/HEP as well as the cooperation of the staff at JLab and Fermilab. Steve Virostek and Mike Dickenson of LBNL and Ben Ogert of Fermilab have been particularly helpful.

\section{REFERENCES}

[1] M. Zisman, The Muon Ionization Cooling Experiment (MICE), this conference.

[2] Y. Torun, et. al., The MTA Facility and Instrumentation, this conference.

[3] R. A. Rimmer, Fabrication of the $201 \mathrm{MHz}$ MICE prototype cavity, Proceedings of PAC05, June 2005, Knoxville, TN (2005). 